\title{
Effect of intermittent inspiratory leaks on measurement of lung clearance index using nitrogen and sulfur hexafluoride
}

\section{To the Editor:}

Leaks during inert gas washout are a major potential source of measurement error in multiple-breath washout (MBW) testing. Visible leaks require exclusion of the whole test repeat [1], whilst undetected leaks may lead to significant errors in estimation of washout indices such as lung clearance index (LCI) and functional residual capacity (FRC) [2]. A recent study attempted to assess the impact on FRC and LCI of leaks of varying magnitude and type using clinically generated data modified to replicate the impact of leaks [2]. This approach is limited by the source data and the assumption that all leaked gas is mixed evenly. The authors, however, showed that the impact of expiratory leaks during nitrogen $\left(\mathrm{N}_{2}\right) \mathrm{MBW}$ was dependent on their size, duration and the point at which they occurred in washout. In contrast, inspiratory leaks invariably led to substantial increases in both FRC and LCI as a result of the introduction of fresh tracer gas into the lungs (room air, containing $80 \% \mathrm{~N}_{2}$ ). The size of leaks being modelled, however, was substantial (10-50\% of tidal volume) and at a level that it is hoped should be visible during testing. Smaller leaks may well be common but may not be visible, yet in the case of $\mathrm{N}_{2}$ washout, may still cause significant inaccuracy in washout indices. Given the recognised differences in sulfur hexafluoride $\left(\mathrm{SF}_{6}\right)$ and $\mathrm{N}_{2}$ washouts, we were interested to discover what level of inspiratory leak would have negligible impact on $\mathrm{LCIN}_{2}$ and to compare this to $\mathrm{LCISF}_{6}$, where small leaks of inspired air do not introduce new tracer gas into the lungs. In order to do this, we have used a recently described mathematical lung model of wash-in-washout that allows a more detailed interpretation of leak impact in situations of minimal leak.

A previously developed computer simulation of gas ventilation and transport in the airway tree was used to model MBW (model $\mathrm{M}$ in [3]). To simulate bronchoconstriction, we applied constrictions $(80 \%$ reduction in diameter) to $0 \%, 10 \%$ and $20 \%$ of the small airways (those originally between 1 and $2.5 \mathrm{~mm}$ in diameter) in the network model. Constricted airways were selected uniformly at random once for each case $(10 \%$ and $20 \%$ ), and that airway tree generated was then used to simulate MBW for every combination of leak fraction and start time.

A leak was simulated by modifying the measured flow rate by a factor of (1-LeakFrac) for leak fractions up to $10 \%$. We modelled pre-capillary leaks that lasted for three breaths only (applied to both inspiration and expiration) and these were introduced at the start of inspiration at a given point in washout in each simulation. For $\mathrm{N}_{2}$ leaks, an inert gas concentration of LeakFrac $\times c_{0}$ (where $c_{0}$ is the starting concentration, which for $\mathrm{N}_{2}$ is equivalent to its concentration in air) was introduced for the mouth boundary condition on inspiration to account for $\mathrm{N}_{2}$ inhaled from the room air.

LCI was calculated as the interpolated number of lung turnovers to reach an end-tidal concentration of $c_{\mathrm{et}}=0.025 c_{0}$. Physiological parameters of the model were FRC $3 \mathrm{~L}$, tidal volume $1 \mathrm{~L}$, airway dead space $0.12 \mathrm{~L}$ and upper-airway dead space $0.05 \mathrm{~L}$.

The baseline model predictions were $\operatorname{LCIN}_{2}=\{5.0,6.2,8.2\}$ and $\operatorname{LCISF}_{6}=\{5.2,6.4,8.4\}$ for $0 \%, 10 \%$ and $20 \%$ of the small airways constricted, respectively. Leaks typically altered measurements made with $\mathrm{N}_{2}$ more

@ERSpublications

Even small air leaks can have a significant impact on LCI measured using $\mathrm{N}_{2}$. This is particularly the case for leaks that occur towards the end of washout. In contrast, leaks generally have a much smaller impact on LCI measured by $\mathrm{SF}_{6}$. http://ow.ly/az7b30lG5Ku

Cite this article as: Whitfield CA, Jensen OE, Horsley A. Effect of intermittent inspiratory leaks on measurement of lung clearance index using nitrogen and sulfur hexafluoride. ERJ Open Res 2018; 4: 00132-2018 [https://doi.org/10.1183/23120541.00132-2018]. 



FIGURE 1 Contour plot showing \% change in lung clearance index (LCI) due to leaks for the case when $10 \%$ of the small airways are constricted. The shading indicates magnitude of the change (from light to dark) and the labelled contour lines also show the direction of change (positive indicating an increase and negative a decrease). This was plotted using a range of leak fractions and start times using a) nitrogen and b) sulfur hexafluoride washout, where each dot indicates a single simulation. Note the differences in scale with a) $5 \%$ and b) $0.5 \%$ change between contour lines.

severely than those with $\mathrm{SF}_{6}$, as the extra inspired $\mathrm{N}_{2}$ during washout affects both FRC measurement and washout duration.

Changes in LCI of $>7.5 \%$ were simulated for $\mathrm{N}_{2}$ leaks only when introduced later in washout (figure 1a), reaching over $25 \%$ for a $10 \%$ leak fraction beginning in the final $10 \%$ of washout. Notably, even very small leaks (1-2\% leak fraction) sustained for only three breaths were enough to cause clinically significant increases in $\operatorname{LCIN}_{2}(>7.5 \%)$ if introduced late in the washout. This is caused by the extra $\mathrm{N}_{2}$ inhaled from room air prolonging the washout by preventing the end-tidal concentration from reaching $2.5 \%$ of the initial concentration as early as it would have done otherwise.

Leaks during $\mathrm{SF}_{6}$ washout affect LCI only via the measurement of $\mathrm{FRC}$ and behave the same as the intermittent expiratory-only leaks as characterised by LENHERR et al. [2], increasing LCISF $_{6}$ if introduced early in the washout and decreasing $\mathrm{LCISF}_{6}$ if introduced late (figure 1b). The maximal observed change in LCISF $_{6}$ was $4 \%$ (10\% leak fraction at start of washout). Leaks in $\mathrm{N}_{2}$ show the reverse effect, showing a much larger magnitude effect later in washout for all cases simulated. This effect was diminished in models with greater bronchoconstriction, as most of the extra $\mathrm{N}_{2}$ introduced was washed in and out of better ventilated lung regions more quickly than resident $\mathrm{N}_{2}$ was washed from the constricted regions. However, with $20 \%$ of small airways constricted, there remained increases of up to $19 \%$ in $\operatorname{LCIN}_{2}$ (simulated for a $10 \%$ leak fraction starting at the final breath of washout). The relative change in LCIsF $_{6}$ due to leaks was not significantly changed from figure $1 \mathrm{~b}$ in either the case of $0 \%$ or $20 \%$ of small airways constricted.

The data from this study complement those from the earlier study by LENHERR et al. [2], also published in ERJ Open Research. The main differences are that we have concentrated on the combined effect of inspiratory and expiratory leaks, and used a sophisticated computer lung model to look at the impact of minor leaks (three breaths, up to a maximum of $10 \%$ tidal volume) on washout outcomes. Using this approach, we have shown that LCI measurement is exquisitely sensitive to inspiratory air leaks during $\mathrm{N}_{2}$ MBW, even when these may be too small to pick up from watching the MBW trace. This is particularly relevant for leaks that occur very late in washout, where a leak of just $2 \%$ of tidal volume was predicted to cause up to an $18 \%$ increase in $\operatorname{LCIN}_{2}$. We have also seen that very small leaks occurring at the start of washout may not have a detectable effect on LCI. Furthermore, the impact of leaks is diminished in those with more severe ventilation heterogeneity since the inspired (leaked) tracer gas is preferentially washed in and then out of the best ventilated lung regions. In all cases, in contrast to the situation described here for nitrogen $\mathrm{MBW}$, washout of $\mathrm{SF}_{6}$ was barely affected by small leaks, being changed by only the small volume of additional air introduced. Unrecognised leaks may contribute to the differences between clinically measured $\mathrm{SF}_{6}$ and $\mathrm{N}_{2}$ washout indices, which can be very substantial $[4,5]$. This study emphasises the need for experienced staff to be highly vigilant when carrying out MBW testing. Since it may be impossible to always be certain that there has been no minimal leak (particularly at the level modelled here), it also illustrates an additional advantage of $\mathrm{SF}_{6}$ as a washout gas.

Carl A. Whitfield $\oplus^{1,2}$, Oliver E. Jensen ${ }^{2}$ and Alex Horsley $\oplus^{1}$

${ }^{1}$ Division of Infection, Immunity and Respiratory Medicine, University of Manchester, Manchester, UK.

${ }^{2}$ School of Mathematics, University of Manchester, Manchester, UK. 
Correspondence: Carl A. Whitfield, Division of Infection, Immunity and Respiratory Medicine, University of Manchester, Education and Research Centre, Wythenshawe Hospital, Manchester, M23 9LT, UK. E-mail: carl.whitfield@physics.org

Received: Aug 102018 | Accepted: Aug 302018

Conflict of interest: C.A. Whitfield reports receiving grants from the UK Medical Research Council during the conduct of the study. O.E. Jensen has nothing to disclose. A. Horsley reports receiving grants from the National Institute for Health Research, during the conduct of the study; and reports grants from the Cystic Fibrosis Foundation and Cystic Fibrosis Trust, and personal fees from Vertex Pharmaceuticals, Celtaxys and Boehringer Ingelheim, and has a collaboration agreement with Innovision ApS, outside the submitted work.

Support statement: Funding was received from the Medical Research Council (Skills Development Fellowship to C.A. Whitfield, grant number MR/R024944/1) and the National Institute for Health Research (CS012-13). Funding information for this article has been deposited with the Crossref Funder Registry.

\section{References}

1 Robinson PD, Latzin P, Verbanck S, et al. Consensus statement for inert gas washout measurement using multipleand single-breath tests. Eur Respir J 2013; 41: 507-522.

2 Lenherr N, Ramsey KA, Jost K, et al. Leaks during multiple-breath washout: characterisation and influence on outcomes. ERJ Open Res 2018; 4: 00012-2017.

3 Whitfield CA, Horsley A, Jensen OE. Modelling structural determinants of ventilation heterogeneity: a perturbative approach. bioRxiv 2018; 329961.

4 Jensen $\mathrm{R}$, Stanojevic S, Gibney $\mathrm{K}$, et al. Multiple breath nitrogen washout: a feasible alternative to mass spectrometry. PLoS One 2013; 8: e56868.

5 Bell A, Lawrence PJ, Singh D, et al. Feasibility and challenges of using multiple breath washout in COPD. Int J Chron Obstruct Pulmon Dis 2018; 13: 2113-2119. 